\title{
A Three-Channel Model for Generating the Vestibulo-Ocular Reflex in Each Eye
}

\author{
LAURENCE R. HARRIS, ${ }^{a}$ KARL A. BEYKIRCH,${ }^{b}$ AND MICHAEL FETTER ${ }^{c}$ \\ ${ }^{a}$ Department of Psychology, York University, Toronto, Canada \\ ${ }^{b}$ Department of Neurology, University of Tübingen, Tübingen, Germany \\ ${ }^{c}$ Department of Neurologie, Klinikum Karlsbad-Langensteinbach, \\ Karlsbad-Langensteinbach, Germany
}

KEYWORDS: vestibulo-ocular reflex; three-dimensional eye movements; channels; passive rotation

Coding head movement involves representing the head's velocity and axis of rotation. The neural representation can then be used to inform perceptual and motor processes. An important motor response to head movement is the compensatory eye movements evoked, one component of which is the vestibulo-ocular reflex (VOR). Historically a three-neuron arc has been described as the core of the neural mechanism underlying the generation of the VOR. ${ }^{1,2}$ Such a direct line between sensor (the canals) and effector (the eye muscles) implies independent processing of the geometric components of the three-dimensional VOR. ${ }^{3}$ A more flexible and robust representation of the movement involves an interactive process in which the activity coding movement in each direction is interpreted in the context of the activity of the others. Many sensory attributes are coded by the activity of a small set of channels, ${ }^{4}$ and the closely constrained three-dimensional movement of the head could be efficiently represented by such a system. Psychophysical methods have been developed to investigate channel systems among which is adaptation. After adapting the response to a particular stimulus, the effect on the responses to closely related stimuli can often reveal a channel-coding system..${ }^{5,6}$ Here we use an adaptation technique to provide evidence for a three-channel model underlying the representation of head rotation and generating the vestibulo-ocular reflex of each eye. These channels are conceptually different from those proposed for coding head velocity ranges, ${ }^{7}$ as discussed elsewhere. ${ }^{8}$

Address for correspondence: Laurence R. Harris, Department of Psychology, York University, 4700 Keele Street, Toronto, Ontario M3J 1P3, Canada. Voice: 416-736-2100, ext. 66108; fax: 416-736-5814.

harris@yorku.ca

Ann. N.Y. Acad. Sci. 956: 537-542 (2002). () 2002 New York Academy of Sciences. 


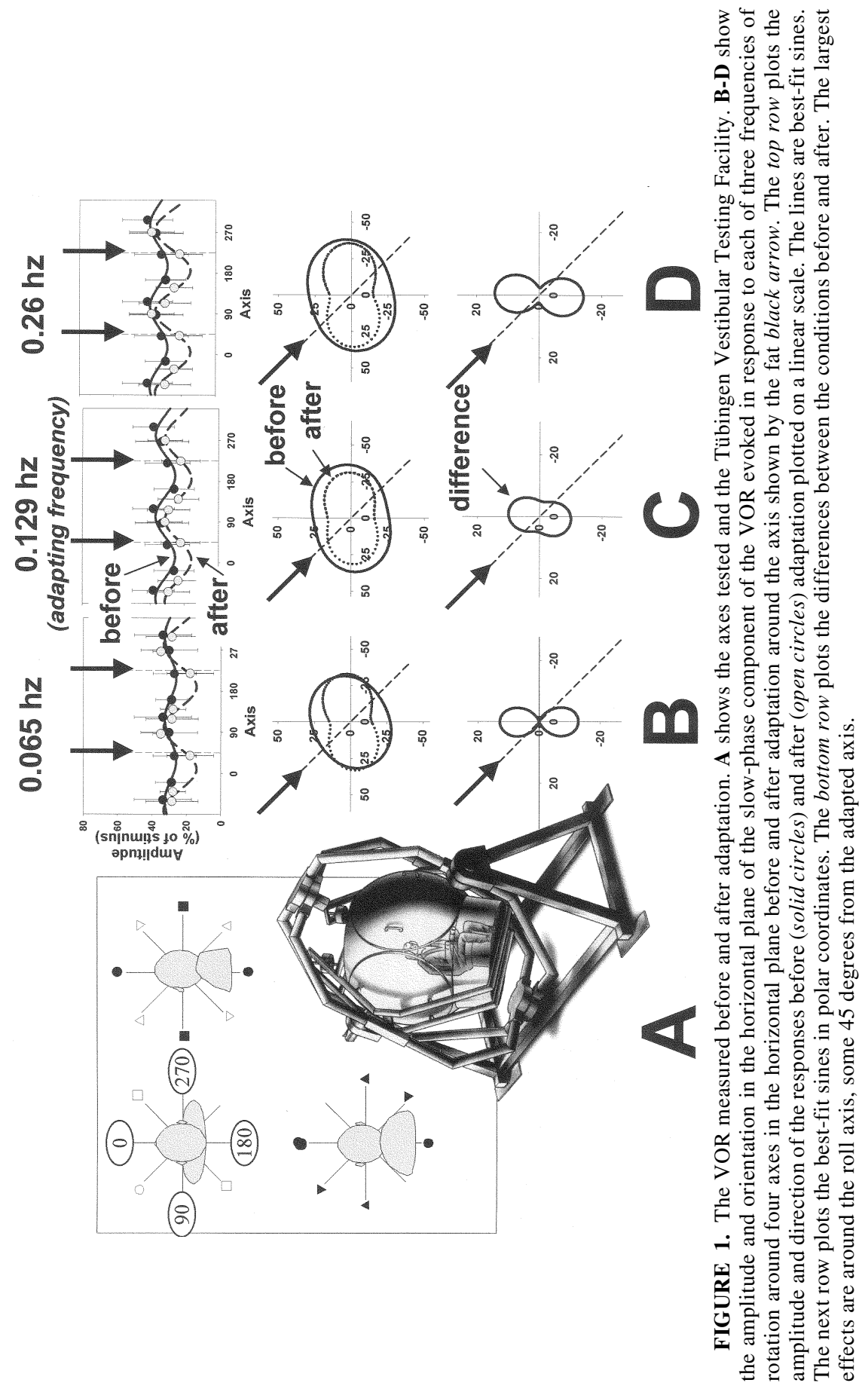




\section{METHODS}

The experiment was run in two sessions separated by at least two days. The first session evaluated the eye movements evoked by rotation around eight test axes. Using the Tübingen Vestibular Research Stimulator and starting from upright, we rotated seven subjects in yaw, pitch, roll and axes 45 degrees in between (FIG. 1A) in the dark and measured the evoked eye movements with $3-\mathrm{D}$ scleral search coils on the left eye. We used $100 \mathrm{sec}$ of sum-of-sines $(0.032,0.065,0.13,0.26 \mathrm{~Hz}, \pm 20 \mathrm{de}-$ grees). In the second session, subjects were adapted to subject-stationary vision viewed at $1 \mathrm{~m}$ during physical rotation $(0.13 \mathrm{~Hz} ; \pm 100$ degrees $)$ in the plane of the right-anterior, left-posterior (RALP) canal-pair. Under these conditions the vestibulo-ocular reflex is suppressed ${ }^{9}$ and the reduced gain persists when performance is measured subsequently in darkness. ${ }^{10}$ After the 30-minute adaptation procedure, eye coils were placed on the left eye, and the VOR evoked by rotation about the test axes measured in the dark. The data analysis method has been described elsewhere. ${ }^{11}$

\section{RESULTS}

The slow-phase component of the VOR induced by rotation before adaptation was not always aligned with the stimulating axis and showed a variation in amplitude and deviation that varied from axis to axis ${ }^{11}$ (FIG. 1B-D [solid circle and solid lines] and FIG. 2 ["pre"]). After visually driven VOR gain reduction by stabilized vision around the RALP axis, the response in the dark to rotation around the adapting axis (indicated by thick arrows in FIG. 1) was reduced. However, changes in both amplitude and alignment were also seen in response to rotation around other axes, most notably roll. The differences between the before and after conditions are illustrated in the bottom row of FIG. 1 B-D: clearly the largest effect is in response to roll rotation. Notice that the response to pitch rotation, which, like roll, was only 45 degrees away from the adaptation axis, was not significantly altered.

\section{MODELING}

The data were modeled with a three-channel model to code the orientation and velocity of head rotation. Each of the three channels of this model represents a component of the movement. They are not based on any anatomical features but are proposed simply as an information-processing system. The head rotation is represented as a three-dimensional vector of a length representing velocity. This vector is then projected orthogonally onto each of the "channels." Each channel has a gain by which the projected component is multiplied and an orientation. To recover the head movement, the activity in the three channels are taken as the coordinates. The output is the vector sum of the three channels' activity.

In order to model the VOR before any adaptation, the orientations and gains of the three channels were configured as free variables and the output of the model, with a random initial configuration, was compared to the actual response for each subject around each axis. Gain and orientations were varied systematically and the process repeated until the output of the model best matched the data. The orientation 


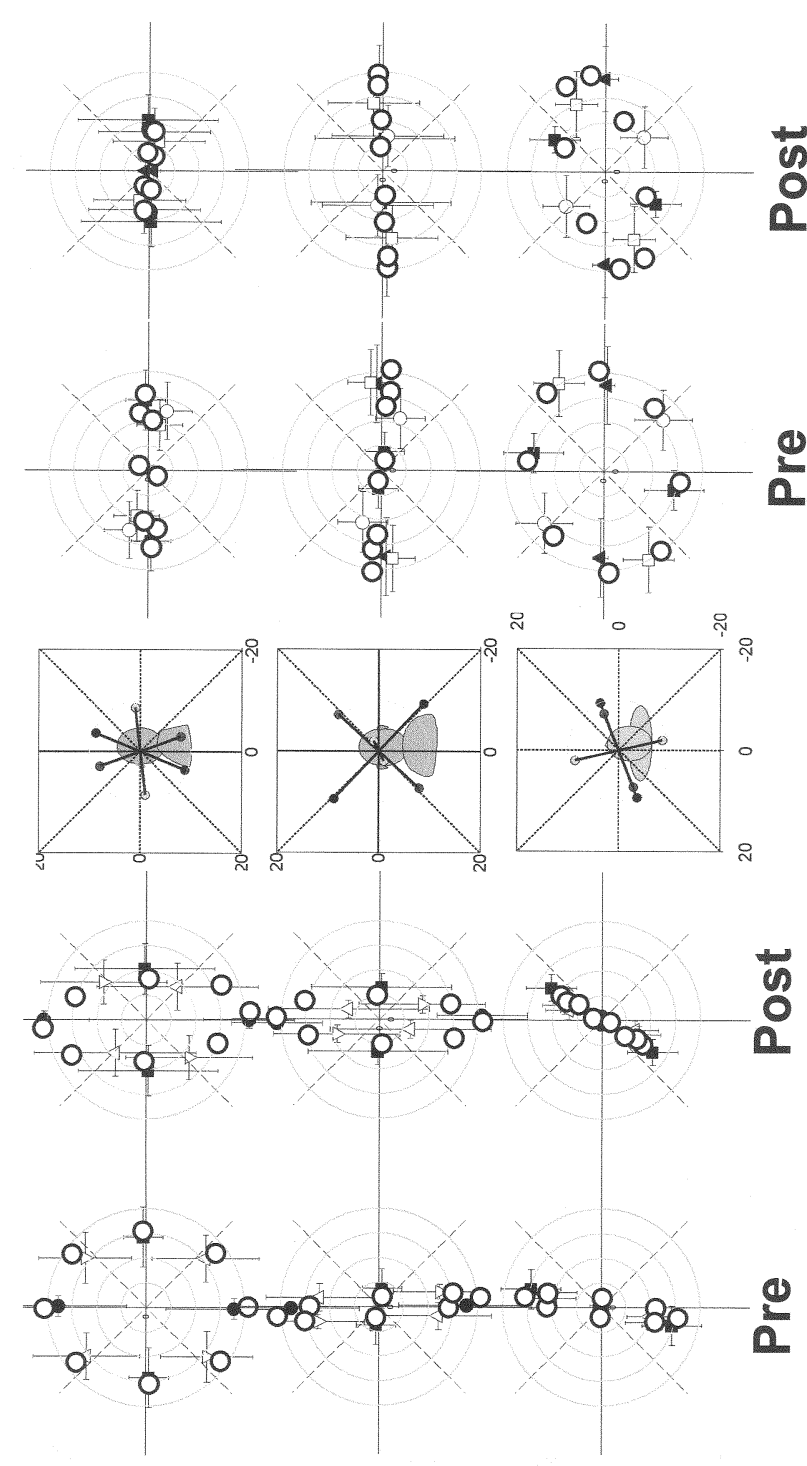

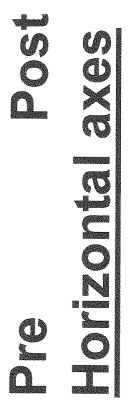

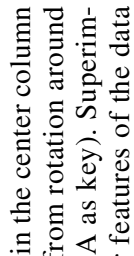

今ั0

的武 I

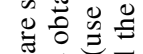

a

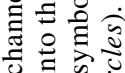

శึ

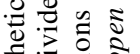

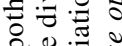

穸卷

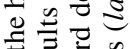

Чั่

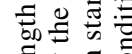

ฮ로유

$2 \sqrt{3}$

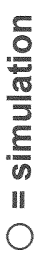

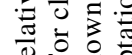

巧 的

สิ

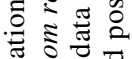

氙 :

:

링

류

จे

过京吾

9
0
$x$
6
$\frac{7}{0}$
\pm
9
0
0

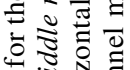

잉 오을

$>$ 过

글

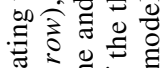

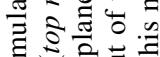

的可元艺

ง 的苛苛

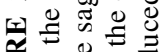

范

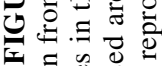

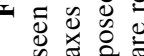


and gains of the set of three channels that optimally reproduced all the major features of the pre-adapt VOR, are shown in FIGURE 2. The prediction of this model of the response to the eight axes used are shown as large open symbols on either side in the columns labelled "pre." There is a good correspondence.

Next the model was "adapted." First the relative hypothetical activity of each of the channels expected in response to the physical motion component of the adapting stimulus was calculated given the gains and orientations of the channels obtained from the best fit to the pre-adapted data.The relative gains of each channel were then adjusted by an amount proportional to their relative activity during the adaptation experience to produce an "adapted" model. The response of the "adapted" model was simulated by projecting each head rotation onto the "adapted" channels (FIG. 2). The output of the adapted model showed an excellent fit to the adapted data, reproducing all the major features.

The properties of the proposed channels (FIG. 2, center) do not correspond to canal or roll/pitch/yaw coordinates. They are far away from the planes of individual canals, with one close to roll and the others forming an X approximately in Listing's plane, which can be expected to be tilted outwards since the eyes were likely verged in dark at a distance roughly corresponding to the screen. ${ }^{12}$ A separate set of channels is needed for each eye since they are not in a plane orthogonal to the straight ahead but are instead tilted outwards by about 20 degrees.

This study shows that the vestibulo-ocular reflex can be elegantly modeled by a three-channel system. The proposed location of the channels correspond to emerging studies indicating a neural coordinate system involving an axis close to roll ${ }^{13}$ and Listing's plane. ${ }^{14-16}$

\section{ACKNOWLEDGMENTS}

This work was supported by the Deutsche Forschungsgemeinschaft; Natural Science \& Engineering Council (NSERC), Canada; and the Centre for Research in Earth and Space Technology (CRESTech), Ontario.

\section{REFERENCES}

1. Lorente DE Nó, R. 1933. Vestibulo-ocular reflex arc. Ann. Neurol. Psychiat. 30: 245-291.

2. Szentágothai, J. 1950. The elementary vestibulo-ocular reflex arc. J. Neurophysiol. 13: 395-407.

3. Vilis, T. \& D. Tweed.1988. A matrix analysis for a conjugate vestibulo-ocular reflex. Biol. Cybernet. 59: 237-245.

4. Blum, B. 1991. Channels in the Visual Nervous System: Neurophysiology, Psychophysics and Models. Freund. London.

5. Campbell, F.W. \& R.W. Tegeder. 1991. A survey of channels and challenges, of information and meaning. In Channels in the Visual Nervous System: Neurophysiology, Psychophysics and Models. B. Blum, Ed.: 1-10. Freund. London.

6. Graham, N. 1989. Visual Pattern Analyzers. Oxford University Press. Oxford.

7. Lisberger, S.G., F.A. Miles, et al. 1983. Frequency-selective adaptation: evidence for channels in the vestibulo-ocular reflex? J. Neurosci. 3: 1234-1244.

8. HARRIS, L.R. 1997. The coding of self motion. In Computational and Psychophysical Mechanisms of Visual Coding. L.R. Harris \& M. Jenkin, Eds.: 157-183. Cambridge University Press. Cambridge. 
9. BARNES, G.R. 1982. Visual factors affecting suppression of the vestibulo-ocular reflex. In Functional Basis of Ocular Motility Disorders. G. Lennerstrand et al. Eds.: 387389. Pergamon. Oxford and New York.

10. Berthoz, A. \& G. Melvill Jones. 1985. Adaptive Mechanisms in Gaze Control. Elsevier. New York.

11. HARRIS, L.R. \& K. BEYKIRCH, et al. 2001. The visual consequences of deviations in the orientation of the axis of rotation of the human vestibulo-ocular reflex. Vision Res. 41: 3271-3281.

12. MoK, D. \& A. Ro, et al. 1992. Rotation of Listing's plane during vergence. Vision Res. 32: 2055-2064.

13. Crawford, J.D. \& W. CAdera, et al. 1991. Generation of torsional and vertical eye position signals by the interstitial nucleus of Cajal. Science 252: 1551-1553.

14. CRAWFORD, J.D. \& T. VILIS. 1991. Axes of eye rotation and Listing's law during rotations of the head. J. Neurophysiol. 65: 407-423.

15. Smith, M.A. \& J.D. CRAWFORD. 1998. Neural control of rotational kinematics within realistic vestibuloocular coordinate systems. J. Neurophysiol. 80: 2295-2315.

16. Smith, M.A. \& J.D. CRAWFord. 2001. Self-organizing task modules and explicit coordinate systems in a neural network model for 3-D saccades. J. Comput. Neurosci. 10: $127-150$. 